\title{
Effects of a pre-inflation radiation-dominated epoch to CMB anisotropy
}

\author{
I-Chin Wang ${ }^{1}$ and Kin-Wang $\mathrm{Ng}^{2}$ \\ ${ }^{1}$ Department of Physics, National Cheng Kung University, \\ Tainan, Taiwan 701, R.O.C. \\ ${ }^{2}$ Institute of Physics, Academia Sinica, \\ Taipei, Taiwan 115, R.O.C.
}

(Dated: October 12, 2007)

\begin{abstract}
We consider that the pre-inflation era is radiation-dominated, transiting smoothly to the inflationary era. We work out in detail the dynamics of inflaton fluctuations across the phase transition and the proper choices of initial vacuum states. It is found that this phase transition can suppress longwavelength quantum fluctuations of inflaton. This may attribute to the large-scale CMB anisotropy a lower power than predicted in the standard $\Lambda$ CDM model. In constraining this transitional effect by WMAP anisotropy data, we use the WMAP best-fit scale-invariant $\Lambda$ CDM model with the density power spectrum replaced by the one found in this work. We find that the transition occurs at least about 10 e-folds before the comoving scales comparable to our present horizon size cross the Hubble radius during inflation.
\end{abstract}

PACS numbers: $98.80 . \mathrm{Cq}, 98.70 . \mathrm{Vc}, 98.80 . \mathrm{Es}$

\section{INTRODUCTION}

The inflationary model can explain the observed spatially flat and homogenous Universe. Moreover, quantum fluctuations during inflation can give rise to primordial density fluctuations with an almost scale-invariant power spectrum, which is consistent with the recent WMAP data on cosmic microwave background anisotropies [1]. Among various cosmological models, the $\Lambda \mathrm{CDM}$ model is a quite good one - it can fit CMB data well. However, the CMB anisotropy measurements made by WMAP have shown that the amplitude of the quadrupole is lower than expected for the $\Lambda \mathrm{CDM}$ model, although the statistical significance of such an anomaly is not large [1]. Recently, there have been many proposed solutions for this anomaly, mostly based on some new ingredients in the generating process of density perturbation [2].

A general assumption of the $\Lambda \mathrm{CDM}$ model is that it considers only a vacuum-dominated inflationary epoch, and that it does not consider the possibility of a pre-inflation era. For example, a radiation-dominated era has taken place before inflation. In fact, this phase transition has been proposed in the context of spontaneous symmetry breaking to provide with a mechanism for inflation [3]. In this paper, we will take into account the effect of a pre-inflation radiation-dominated era. As expected, if inflation lasts very long such that the present Universe only occupies a tiny portion of the inflated region, the effect of the phase transition will be negligible. However, if it happens that the present Universe is just comparable to the size of the inflated region, the pre-radiation era may leave imprints on large-scale structures of the Universe. Here we will study the effect of the phase transition to the large-scale CMB anisotropy. Recently, this effect has been discussed by Powell and Kinney [4], who considered large-wavelength inflaton fluctuations in the pre-inflation radiation-dominated era and found a suppression of power on large scales as a result of the choices of vacuum states. (See also Ref. [5] for a similar consideration.) In addition, Contaldi et al. in Ref. [2] have found a similar effect caused by a pre-inflation epoch that is dominated by the kinetic energy of the inflation. However, the issues about the dynamics of inflaton fluctuations across the phase transition and the proper choices of initial vacuum states have not been fully investigated. Here, we will study these issues in detail.

Let us simply consider that the Universe has two components during the phase transition - one is radiation and the other is vacuum energy. We assume that the pre-inflationary Universe is in a radiation-dominated phase and then the Universe evolves to a vacuum-energy-dominated or de Sitter phase. Although the solution for this transition has been obtained for many years [6, 7, 8, 9], there is no attempt in varying the amounts of radiation and vacuum energies of the Universe to see the effect on CMB anisotropy. This serves a way to test the existence of an unknown pre-inflation phase. Also, it provides a constraint on the period of inflation which needs to be consistent with the observed CMB anisotropy spectrum under such a pre-inflation condition.

In this paper, we use Vilenkin and Ford's transition solution [6] to compute the power spectrum of quantum fluctuations of the inflaton field. Since the pre-inflation phase is radiation-dominated, we use the radiation-dominated solution to determine the boundary conditions. This allows us to set the form of the seed for the quantum fluctuations before the de Sitter phase begins. By introducing this pre-inflation initial condition, we can calculate the power spectrum of the horizon-crossing modes near the transition region. Then we input the power spectrum to the CMBFAST numerical code [10] to generate the CMB anisotropy power spectrum. We vary the amounts of radiation and vacuum 
energies and tune the duration of inflation to obtain different results. In order to compare the results with the $\Lambda$ CDM model, we do the chi-square fitting of the generated CMB anisotropy power spectra to the WMAP data and calculate the chi-square values.

In next section, we will derive the mode equation of the quantum fluctuation during the phase transition. The numerical results for the mode equation and the respective CMB anisotropy power spectra will be given in Sec. III. Sec. IV] is our conclusion.

\section{THE MODEL}

We consider a flat Robertson-Walker metric, with a scale factor $a(t)$ :

$$
d s^{2}=g_{\mu \nu} d y^{\mu} d y^{\nu}=d t^{2}-a^{2}(t) d \vec{y}^{2}
$$

The total energy-momentum tensor is given by

$$
T_{\mu \nu}=T_{\mu \nu}^{(r a d)}+V_{0} g_{\mu \nu}
$$

where $T_{\mu \nu}^{(\mathrm{rad})}$ is the trace-free energy momentum tensor for radiation and $V_{0}$ is constant vacuum energy. Hence, the Einstein evolution equation is

$$
H^{2} \equiv\left(\frac{1}{a} \frac{d a}{d t}\right)^{2}=\frac{\rho}{3 M_{P l}^{2}}
$$

where $M_{P l}=(8 \pi G)^{-1 / 2}=2.436 \times 10^{18} \mathrm{GeV}$ is the reduced Planck mass and $\rho$ is given by

$$
\rho=3 M_{G}^{4}\left(\frac{A}{a^{4}}+B\right),
$$

with $V_{0}=3 B M_{G}^{4}$ of order of the grand unification energy scale. $A$ and $B$ are dimensionless constant parameters. Defining $\Lambda^{2} \equiv M_{G}^{4} / M_{P l}^{2}$, the solution of Eqs. (3]) and (41) is found as [6]

$$
a(t)=\left(\frac{A}{B}\right)^{\frac{1}{4}}[\sinh (2 \sqrt{B} \Lambda t)]^{\frac{1}{2}} .
$$

The asymptotic form in the radiation-dominated phase is

$$
\Lambda t \ll \frac{1}{2} B^{-\frac{1}{2}}, a(t) \sim \sqrt{2} A^{\frac{1}{4}}(\Lambda t)^{\frac{1}{2}}
$$

The asymptotic form in the inflationary phase is

$$
\Lambda t \gg \frac{1}{2} B^{-\frac{1}{2}}, \quad a(t) \sim 2^{-\frac{1}{2}}\left(\frac{A}{B}\right)^{\frac{1}{4}} e^{\sqrt{B} \Lambda t} .
$$

To study the transition from the pre-inflation radiation-dominated phase to inflation, we put Eq. (55) into the equation of motion for the inflaton quantum fluctuation. To a good approximation, we consider the zero-mass case. The equation of motion for the quantum fluctuation of inflaton is given by

$$
\square \varphi(t, \vec{y})=0,
$$

where

$$
\square=-\frac{1}{\sqrt{-g}} \partial_{\mu}\left(\sqrt{-g} g^{\mu \nu} \partial_{\nu}\right) .
$$

The Fourier component of $\varphi(t, \vec{y})$ is given by

$$
\phi_{k}(t)=\int \varphi(t, \vec{y}) e^{i \vec{k} \cdot \vec{y}} d \vec{y} .
$$


Put Eq. (10) into Eq. (8), then Eq. (8) becomes

$$
\ddot{\phi}_{k}(t)+3 \frac{\dot{a}}{a} \dot{\phi}_{k}(t)+\left(\frac{k^{2}}{a^{2}}\right) \phi_{k}(t)=0, \dot{\phi}_{k}(t) \equiv \frac{d \phi_{k}(t)}{d t} .
$$

To solve Eq. (11) with $a(t)$ given by Eq. (5), we change the derivative of an independent variable from the time $t$ to the scale factor $a(t)$. As we want to study the effect of the transition around the critical point $a_{c}$, where $a_{c}$ is given by $A / a_{c}^{4}=B$, we rewrite Eq. (11) in terms of $x \equiv a-a_{c}$. Then, Eq. (11) becomes

$$
\left[B\left(x+a_{c}\right)^{4}+A\right] \phi_{k}^{\prime \prime}(x)+\left[4 B\left(x+a_{c}\right)^{3}+\frac{2 A}{x+a_{c}}\right] \phi_{k}^{\prime}(x)+k^{2} \phi_{k}(x)=0 \quad, \quad \phi_{k}^{\prime}(x) \equiv \frac{d \phi_{k}(x)}{d x} .
$$

Note that when $a(t)$ is small, the Universe is in radiation-dominated era. Therefore, we choose the boundary conditions for Eq. (12) as

$$
\begin{array}{r}
\phi_{k}(x)=\frac{1}{a} \frac{1}{\sqrt{2 k}} e^{i k a / \sqrt{A}}, \\
\phi_{k}^{\prime}(x)=\left[-\frac{1}{\sqrt{2 k} a^{2}}+\frac{i \sqrt{k}}{\sqrt{2 A} a}\right] e^{i k a / \sqrt{A}} .
\end{array}
$$

Solving Eq. (12) with above boundary conditions gives the solution for a particular $k$ mode. The relation $k=a H$ determines the value of $k$ for the mode that crosses the horizon at $a=a_{k}$, where $H$ is the Hubble parameter given by Eq. (3). This gives from Eq. (3) that

$$
\frac{k}{\Lambda}=\left(\frac{A}{a_{k}^{2}}+B a_{k}^{2}\right)^{\frac{1}{2}}
$$

Since we are concerned with the $k$-modes that leave the horizon shortly after inflation begins, we will consider only the solutions with $k \geq k_{c}$, where $k_{c}=\Lambda \sqrt{2 B} a_{c}$. The power spectrum of the quantum fluctuations of the inflation is then given by

$$
P_{k}^{1 / 2} \propto k^{3 / 2} \phi_{k}\left(x_{k}\right)
$$

where $x_{k}=a_{k}-a_{c}$. In the following section, we compute power spectra $P_{k}^{1 / 2}$ produced when and after inflation begins $(x \geq 0)$ by numerically solving Eq. (12) for different values of $A$ and $B$. Then, we put these results into the CMBFAST code [10] to generate the CMB anisotropy power spectra.

\section{NUMERICAL RESULTS}

So far, we have not specified the values of the parameters $A$ and $B$. Before doing the numerical calculation, let us assess the possible range of the values of $A$ and $B$.

From Eq. (4), the vacuum energy that drives inflation is given by

$$
V_{0}=3 M_{G}^{4} B
$$

Here we consider $V_{0}$ as the potential energy of an inflaton potential $V(\varphi)$ during inflation. The measurements of the CMB anisotropy made by WMAP has put a constraint on $V(\varphi)$ given by [1]

$$
V^{\frac{1}{4}}=0.0265 \epsilon^{\frac{1}{4}} M_{P l},
$$

where the slow-roll parameter

$$
\epsilon \equiv\left(M_{P l}^{2} / 2\right)\left(V^{\prime} / V\right)^{2}<0.033
$$

at $95 \%$ confidence level. Therefore, we have $V_{0} \simeq V$ and thus

$$
B^{\frac{1}{4}}<0.0086 M_{P l} / M_{G} \text {. }
$$


If we take $M_{G}=2.1 \times 10^{16} \mathrm{GeV}$, then $B<1$.

In the following, we choose $B=1$ and $B=0.1$ to calculate the power spectra of inflaton fluctuations $P_{k}$ in Eq. (16). Although we have introduced the slow-roll parameter $\epsilon$, we will assume a pure de Sitter space during inflation and neglect the small slow-roll corrections to the density power spectrum. This would not affect our interest here since the effect of a pre-inflation radiation-dominated epoch is mainly on large angular scales. Then, we use the resulting density power spectrum and the other cosmological parameters in the WMAP best-fit scale-invariant $\Lambda$ CDM model (i.e. the density power spectrum has a scalar spectral index $n_{s}=1$ ) [1] to generate the CMB anisotropy power spectra. Note that while the observation gives the constraint on $B$, the value of $A$ is undetermined. In fact, different values of $A$ introduce different boundary conditions as shown in Eqs. (13) and (14). Therefore, we pick a certain value of $A$ to see how the value affects the CMB anisotropy power spectrum and study under what conditions the results are compatible with present CMB observations. In order to give a quantitative comparison with the CMB data, we use the chi-square fitting, $\chi^{2}=\sum_{\imath, j}\left(D_{\imath}^{b}-T_{\imath}^{b}\right) C_{\imath, j}^{-1}\left(D_{j}^{b}-T_{j}^{b}\right)$, and the chi-square value as a measure, where $D_{\imath}^{b}$ is the measured $\imath$ th band-power, $T_{\jmath}^{b}$ is the theoretical value, and $C_{\imath, \jmath}$ is the width of the error bar in measurement.

We present the following two cases:

(1) $B=1$ with $A=7,5,1,0.1$. In this case, the transition takes place at $a_{c}=1.63-0.56$ with $k_{c} / \Lambda=2.30-0.80$. Figure 1(a) shows the power spectrum of inflaton fluctuations $P_{k}^{1 / 2}$ for each set of $B$ and $A$. It is apparent that the large-scale or small- $k$ power is suppressed by the presence of the pre-inflation radiation-dominated epoch. This suppression is similar to that found in Ref. [4]. However, there are two main differences. For large- $k$ modes, we have assumed a standard inflationary scale-invariant power spectrum with $n_{s}=1$ while they have taken the spectrum with $n_{s}=0.951$. For small-k modes, our spectrum increases monotonically with $k$ whereas their spectrum undergoes oscillations; however, both spectra rapidly approach the inflationary spectrum. This oscillatory behavior, also found by Contaldi et al. in Ref. 2] (the result from an analytic computation as shown in Fig. 1 of their paper) and Enqvist et al. in Ref. [9], is actually an artifact resulted from the discontinuity of Ricci curvature at the abrupt phase transition. This artificial particle production can be avoided by considering for example a $C^{\infty}$ function for the scale factor $a(t)$ [12], as we have worked with in Eq.(5).

Besides, we must be very careful about choosing the initial point $a_{i}$ while doing the numerical calculation. For example, in our case with $B=A=1$, the phase transition point is at $a_{c}=1$. We have obtained a smooth power spectrum (see Fig. 1(a)) by choosing the initial $a_{i}$ to be $a_{i}=0.0001$ (which means that it is in the very early Universe; therefore, it is purely radiation-dominated and also very far from the phase transition point $a_{c}=1$ ). But, if we tune the initial $a_{i}$ to be close to the phase transition point, say $a_{i}=0.3$, the oscillation begins to show up. And if we tune the initial $a_{i}$ closer to the phase transition point, for example, $a_{i}=0.6$, the oscillation becomes larger than that in the $a_{i}=0.3$ case, as shown in Fig. 1(b). This test explains that if the initial $a_{i}$ is not in the proper region (proper region means the radiation-dominated region and that is the right region for the radiation-dominated solution in Eq. (13), while improper region means the phase transition region which is not a radiation-dominated region and therefore the solution for $\phi_{k}(x)$ is not Eq. (13) anymore). This improper choice for the initial $a_{i}$ indeed leads to an oscillating power spectrum. However, if the initial $a_{i}$ is carefully chosen in the proper region, it does not lead to an oscillating power spectrum. Note that here we have used the scale factor $a$ as the evolution variable. If we have used the conformal time, we would have obtained an oscillating power spectrum which approaches the flat inflationary spectrum at large $k$. This explains why the power spectrum obtained by Contaldi et al. in Ref. [2] (see Fig. 2 of their paper) is oscillating although it is obtained from an exact numerical evolution. In their paper, they have indeed used an improper choice of the initial condition which is too close to the phase transition. (They have chosen an initial kinetic energy of the inflaton only about 100 times of the potential energy. Since the kinetic energy of the inflaton decreases as $a^{-6}$, both energies rapidly become comparable. To obtain a correct power spectrum, they should have used a much larger initial kinetic energy.)

The power suppression would affect the CMB anisotropy power spectrum. For a spectrum $P_{k}^{1 / 2}$, we choose a value of $z \equiv k_{0.05} / \Lambda$ corresponding to the physical scale of $0.05 \mathrm{Mpc}^{-1}$ and then input this $P_{k}^{1 / 2}$ into the CMBFAST code to generate the CMB anisotropy power spectrum $C_{l}$. This value of $z$ also corresponds to an exponential expansion with the number of e-folds from the start of inflation given by

$$
N_{z}=\ln \left(a_{k} / a_{c}\right) \simeq \ln z .
$$

The results are shown in Fig. 2. Since the measured low-l multipoles of the CMB anisotropy power spectrum are slightly different in the three-year WMAP data (WMAP3) [13] from the one-year data (WMAP1) 14], we compare the chi-square fitting by using WMAP3 data with that with WMAP1 data. The results are summarized in Tables \ and $[$.

The $\chi^{2}$ value of the $\Lambda$ CDM model with a scale-invariant power spectrum using WMAP3 data is 47.09. From Table [1] we can see that the $\chi^{2}$ value for the model decreases to about 48 as $z$ increases to $3 \times 10^{4}$, corresponding to $N_{z} \simeq 10$ e-folds after the start of inflation. This can be reflected, for example in Fig. 2 b, by those five curves for 
$z=120,300,500,1000,3000$ rising in turn from down to top. As we further increase the value of $z$, the resulting $C_{l}$ approaches to that of the $\Lambda \mathrm{CDM}$ model with a scale-invariant power spectrum. Thus, it can be concluded that the WMAP3 data does not prefer the presence of a pre-inflation radiation-dominated epoch, although it is not excluded. If we assume that the comoving scales comparable to our present horizon size cross the Hubble radius during inflation when there are $N_{c m b} \simeq 60$ e-folds from the end of inflation [15], then the radiation-dominated epoch would take place at least $N=N_{z}+N_{c m b} \simeq 70$ e-folds from the end of inflation.

The case is different when using WMAP1 data since the measured one-year low- $l$ multipoles are slightly smaller than the three-year results. The $\chi^{2}$ value of the $\Lambda$ CDM model with a scale-invariant power spectrum using WMAP1 data is 64.99. From Table $\llbracket$, we can see that the $\chi^{2}$ values for all other models are slightly lower than this value at $z \sim 10^{4}$, corresponding to $N_{z} \simeq 9$ e-folds after the start of inflation. Therefore, the WMAP1 data would, albeit a rather low significance, indicate that the universe would be actually predominated by radiation when $N=N_{z}+N_{c m b} \simeq 69$ e-folds from the end of inflation.

(2) $B=0.1$ with $A=7,5,1,0.1$. The transition takes place at $a_{c}=2.89-1.00$ with $k_{c} / \Lambda=1.29-0.45$. We do not show the power spectrum of inflaton fluctuations $P_{k}$ for this case which is indeed quite similar to the previous case. Figure 3 shows the $C_{l}$ power spectra and their $\chi^{2}$ values are given in Tables III and IV] In Table III, the $\chi^{2}$ value decreases rapidly to about 47 at $z \sim 3000$; however, at large $z$ all models approach to the $\Lambda$ CDM model with a scale-invariant power spectrum. In Table IV] the $\chi^{2}$ values for all the models reach a minimum at $z \sim 1000$, which is lower than the $\chi^{2}$ value for the $\Lambda \mathrm{CDM}$ model with a scale-invariant power spectrum. This corresponds to an inflationary expansion with e-folds of $N_{z} \simeq 7$ after the start of inflation. Because the $\chi^{2}$ values decrease with $z$ in the $B=0.1$ case faster than the $B=1$ case, the anisotropy power spectra are closer to each other and the $B=0.1$ case reaches the standard $\Lambda \mathrm{CDM}$ model at a smaller $z$ value. It means that inflation in the $B=1$ case lasts longer than the $B=0.1$ case in order to be consistent with the CMB anisotropy power spectrum observed today. We have assumed that inflation is driven by a nearly flat potential and thus neglected any spectral index. As we can see in Fig. 3 there is a certain degeneracy in the CMB power spectrum between the variation of the spectral index and the existence of a pre-inflation radiation-dominated era, so a joint likelihood fitting of relevant cosmological parameters to WMAP data should be carried out before drawing any conclusion about pre-inflation physics.

\section{CONCLUSION}

We have shown that the existence of a pre-inflation radiation-dominated phase can affect the CMB anisotropy power spectrum. This transition can give a low quadrupole value by adjusting the values for $A, B$, and $z$. The smaller is the value of $z$, the closer is the comoving scales of the present Universe to the transition when they cross out the horizon during inflation. This manifests as rising up or down for those low- $l C_{l}$ of the $z=120$ spectra in Figs. 2 and 3. Meanwhile, the high- $l C_{l}$, for example, in Fig. $2 \mathrm{~d}$ is significantly lower than the observed values. So, it is unlikely that the inflationary era lasts for only 60 e-folds. As expected, the effect of the radiation phase becomes weaker as the value of $z$ increases. From the three-year WMAP data, using the WMAP best-fit $\Lambda$ CDM model parameters, we have found that the phase transition occurs at least 70 e-folds from the end of inflation. Interestingly, the one-year WMAP data indeed prefers very slightly the pre-inflation radiation-dominated phase taking place as early as about 70 e-folds from the end of inflation. Future CMB observations will provide more severe constraints on this transitional effect, and a joint likelihood fitting to CMB data for studying the degeneracy between the addition of a pre-inflation radiation-dominated era and other relevant cosmological parameters will certainly be necessary.

\section{Acknowledgments}

This work was supported in part by the National Science Council, Taiwan, ROC under the Grant NSC 95-2112-M001-052-MY3.

[1] D. N. Spergel et al., Astrophys. J. Suppl. Ser. 170, 377 (2007).

[2] A. Berera, L.-Z. Fang, and G. Hinshaw, Phys. Rev. D 57, 2207 (1998); G. Efstathiou, Mon. Not. Roy. Astron. Soc. 343, L95 (2003); C. R. Contaldi et al., J. Cosmol. Astropart. Phys. 07 (2003) 2; J. M. Cline, P. Crotty, and J. Lesgourgues, J. Cosmol. Astropart. Phys. 09 (2003) 10; B. Feng and X. Zhang, Phys. Lett. B 570, 145 (2003); S. Tsujikawa, R. Maartens, and R. Brandenberger, Phys. Lett. B 574, 141 (2003); T. Moroi and T. Takahashi, Phys. Rev. Lett. 92, 091301 (2004); W. Lee and L.-Z. Fang, Phys. Rev. D 69, 023514 (2004); Y.-S. Piao, B. Feng, and X. Zhang, Phys. Rev. D 69, 103520 (2004); C. Gordon and W. Hu, Phys. Rev. D 70, 083003 (2004); M. Liguori et al., J. Cosmol. Astropart. Phys. 08 (2004) 11; 
D. Boyanovsky, H. J. de Vega, and N. G. Sanchez, Phys. Rev. D 74, 123007 (2006); C.-H. Wu et al., J. Cosmol. Astropart. Phys. 02 (2007) 6.

[3] See, for example, E. W. Kolb and M. S. Turner, The Early Universe (Addison-Wesley, 1990).

[4] B. A. Powell and W. H. Kinney, Phys. Rev. D 76, 063512 (2007).

[5] N. Kaloper and M. Kaplinghat, Phys. Rev. D 68, 123522 (2003).

[6] A. Vilenkin and L. H. Ford, Phys. Rev. D 26, 1231 (1982).

[7] A. A. Starobinsky, Phys. Lett. B 117, 175 (1982).

[8] A. D. Linde, Phys. Lett. B 116, 335 (1982).

[9] K. Enqvist, K.-W. Ng, and K. A. Olive, Nucl. Phys. B 303, 713 (1988).

[10] U. Seljak and M. Zaldarriaga, Astrophys. J. 469, 437 (1996).

[11] H. V. Peiris and R. Easther, J. Cosmol. Astropart. Phys. 10 (2006) 017.

[12] See, for example, D. J. H. Chung, E. W. Kolb, and A. Riotto, Phys. Rev. D 59, 023501 (1998).

[13] G. Hinshaw et al., Astrophys. J. Suppl. Ser. 170, 288 (2007).

[14] C. L. Bennett et al., Astrophys. J. Suppl. Ser. 148, 1 (2003).

[15] A. R. Liddle and S. M. Leach, Phys. Rev. D 68, 103503 (2003).

\begin{tabular}{|c|c|c|c|c|c|c|c|}
\hline & $z=120$ & $z=300$ & $z=500$ & $z=10^{3}$ & $z=3 \times 10^{3}$ & $z=10^{4}$ & $z=3 \times 10^{4}$ \\
\hline$\chi^{2}(B 1 A 7)$ & 997 & 367.2 & 211.3 & 103 & 57.91 & 49.27 & 47.70 \\
\hline$\chi^{2}(B 1 A 5)$ & 1299 & 443 & 217 & 102 & 57.43 & 49.16 & 47.67 \\
\hline$\chi^{2}(B 1 A 1)$ & 3548 & 449.4 & 209.6 & 98.37 & 56.12 & 48.87 & 47.59 \\
\hline$\chi^{2}(B 1 A 0.1)$ & 1574 & 411.6 & 208.8 & 99 & 56.54 & 48.96 & 47.61 \\
\hline
\end{tabular}

TABLE I: Using WMAP3 data to the chi-square fitting of the CMB anisotropy power spectra for $B=1$ and $A=7,5,1,0.1$ with different $z$ values. Note that the chi-square value of the $\Lambda$ CDM model with a scale-invariant power spectrum is 47.09 .

\begin{tabular}{|c|c|c|c|c|c|c|c|}
\hline & $z=120$ & $z=300$ & $z=500$ & $z=10^{3}$ & $z=3 \times 10^{3}$ & $z=10^{4}$ & $z=3 \times 10^{4}$ \\
\hline$\chi^{2}(B 1 A 7)$ & 639.50 & 236.37 & 146 & 85.45 & 65.93 & 64.47 & 64.72 \\
\hline$\chi^{2}(B 1 A 5)$ & 822.23 & 280.6 & 147.16 & 84.83 & 65.81 & 64.48 & 64.74 \\
\hline$\chi^{2}(B 1 A 1)$ & 2238 & 283.35 & 142.47 & 82.90 & 65.44 & 64.50 & 64.77 \\
\hline$\chi^{2}(B 1 A 0.1)$ & 992 & 261.4 & 142.2 & 83.51 & 65.55 & 64.49 & 64.75 \\
\hline
\end{tabular}

TABLE II: The same as in Table @ but using WMAP1 data. Note that the chi-square value of the $\Lambda$ CDM model with a scale-invariant power spectrum is 64.99 .

\begin{tabular}{|c|c|c|c|c|c|c|c|}
\hline & $z=120$ & $z=300$ & $z=500$ & $z=10^{3}$ & $z=3 \times 10^{3}$ & $z=10^{4}$ & $z=10^{5}$ \\
\hline$\chi^{2}(B 0.1 A 7)$ & 59 & 56.88 & 52.82 & 49.83 & 47.97 & 47.43 & 47.11 \\
\hline$\chi^{2}(B 0.1 A 5)$ & 67.3 & 56.9 & 52.59 & 49.66 & 47.89 & 47.32 & 47.11 \\
\hline$\chi^{2}(B 0.1 A 1)$ & 80.08 & 56.19 & 51.78 & 49.08 & 47.67 & 47.23 & 47.09 \\
\hline$\chi^{2}(B 0.1 A 0.1)$ & 77.98 & 55.18 & 51 & 48.63 & 47.49 & 47.2 & 47.08 \\
\hline
\end{tabular}

TABLE III: $\chi^{2}$ values for $B=0.1$ and $A=7,5,1,0.1$, using WMAP3 data. 


\begin{tabular}{|c|c|c|c|c|c|c|c|}
\hline & $z=120$ & $z=300$ & $z=500$ & $z=10^{3}$ & $z=3 \times 10^{3}$ & $z=10^{4}$ & $z=10^{5}$ \\
\hline$\chi^{2}(B 0.1 A 7)$ & 67.13 & 65.78 & 64.8 & 64.49 & 64.66 & 64.87 & 64.99 \\
\hline$\chi^{2}(B 0.1 A 5)$ & 69.68 & 65.75 & 64.76 & 64.49 & 64.68 & 64.87 & 64.98 \\
\hline$\chi^{2}(B 0.1 A 1)$ & 74.51 & 65.49 & 64.6 & 64.50 & 64.75 & 64.91 & 64.99 \\
\hline$\chi^{2}(B 0.1 A 0.1)$ & 73.54 & 65.21 & 64.5 & 64.51 & 64.80 & 64.95 & 64.99 \\
\hline
\end{tabular}

TABLE IV: $\chi^{2}$ values for $B=0.1$ and $A=7,5,1,0.1$, using WMAP1 data.

FIG. 1 (a)

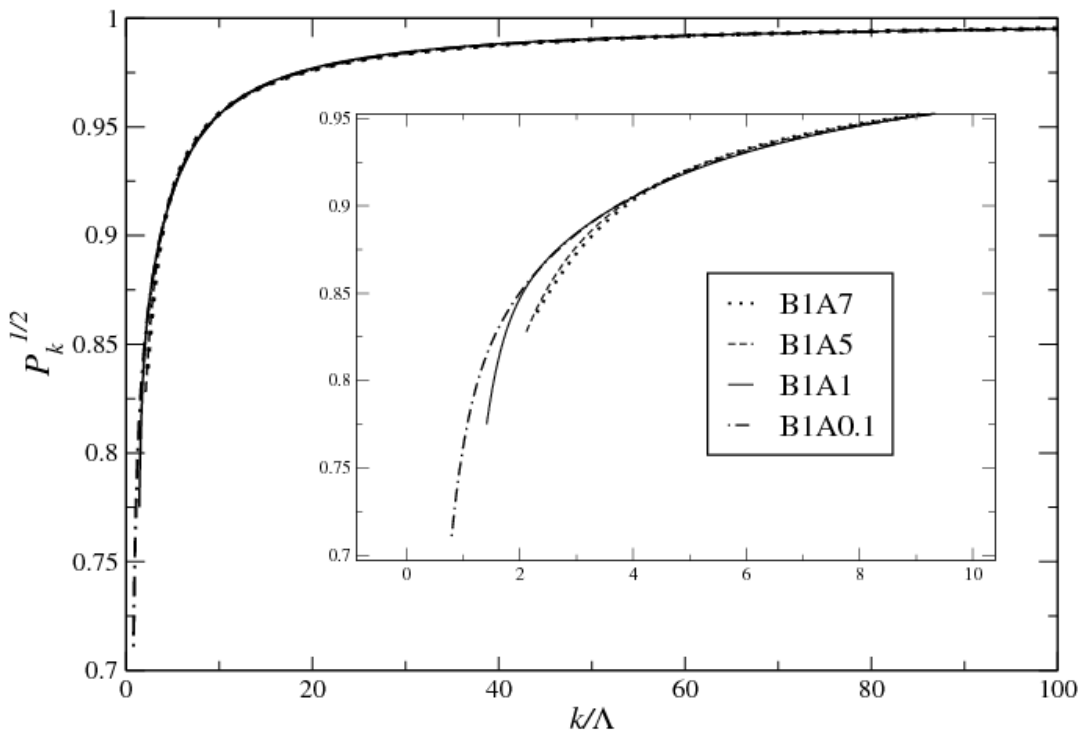

FIG. 1 (b)

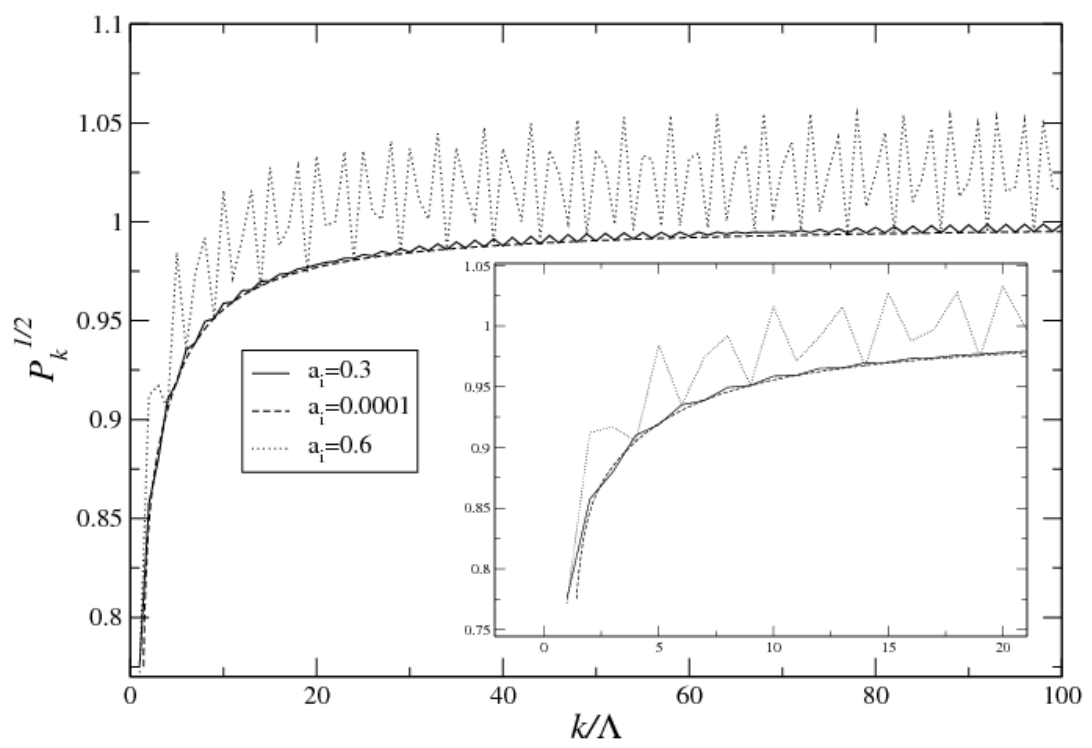

FIG. 1: (a) Normalized power spectra of inflaton fluctuations with $A=7,5,1,0.1$ for a fixed $B=1$. Inserted panel shows the spectra for small $k$. All curves start with $k=k_{c}$ corresponding to the $k$-mode that leaves the horizon at the start of inflation. (b) Normalized power spectra of inflaton fluctuations with initial $a_{i}=0.001,0.3,0.6$ for the case with $B=A=1$. The phase transition point in this case is $a_{c}=1$. 
FIG. 2 (a) B1A7

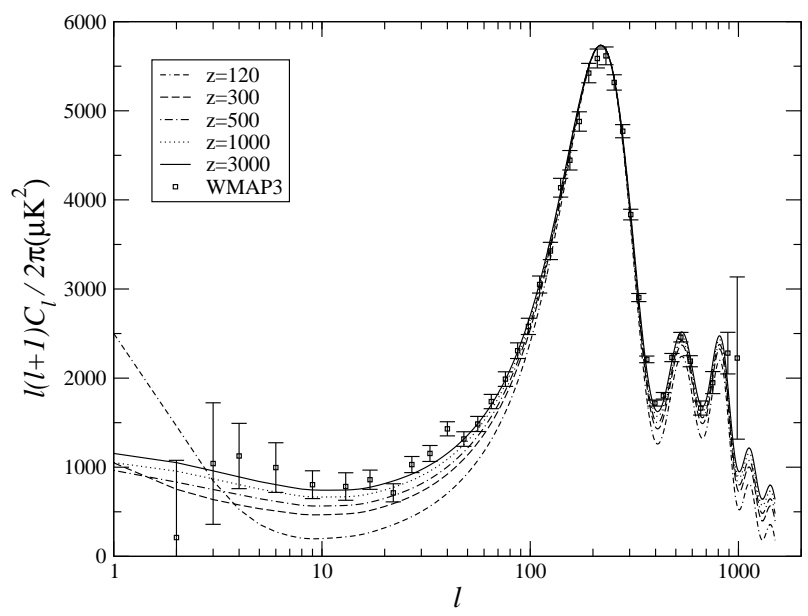

FIG. 2 (c) B1A1

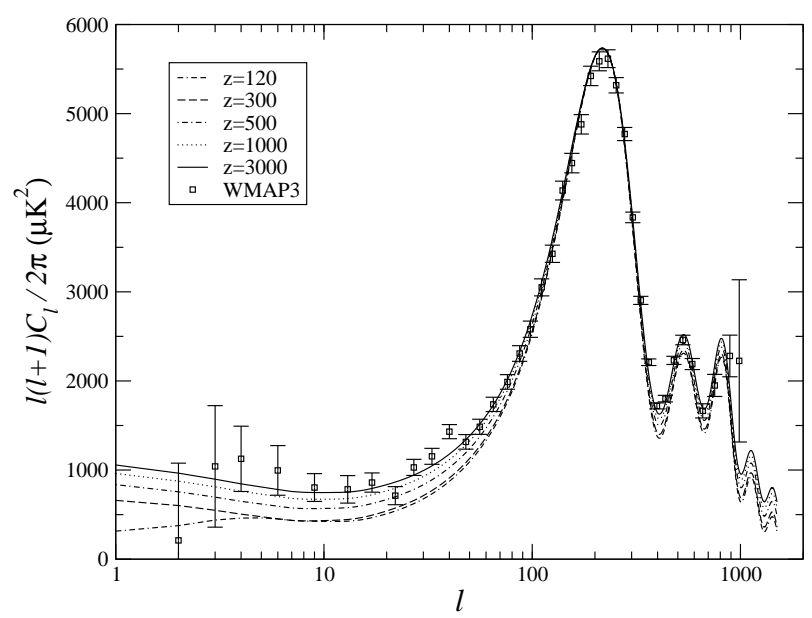

FIG. 2 (b) B1A5

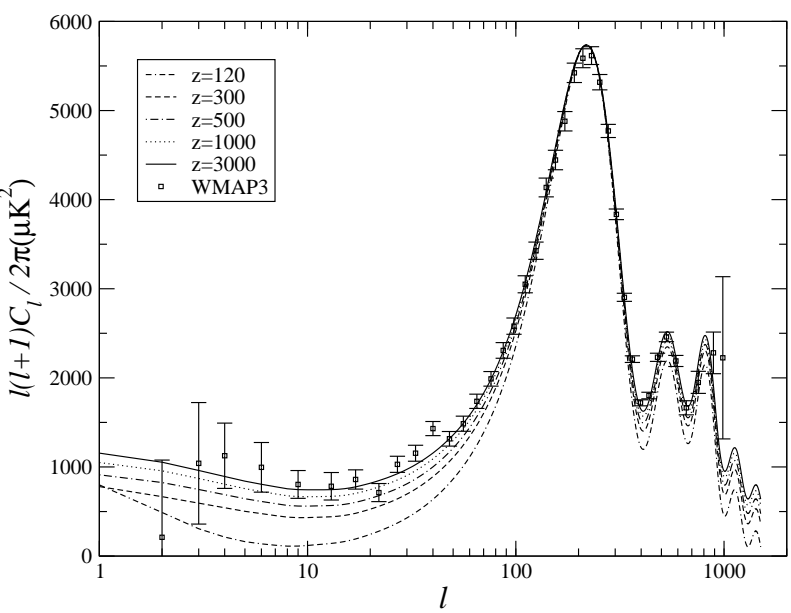

FIG. 2 (d) $\quad$ B1A0.1

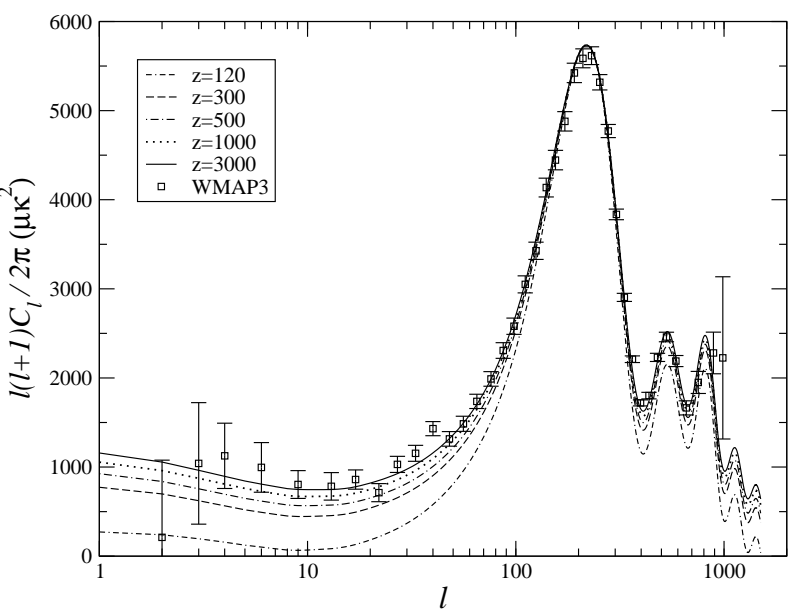

FIG. 2: CMB anisotropy power spectra for $B=1$ and $A=7,5,1,0.1$. In each panel, the curves are induced by the $P_{k}$ with $z \equiv k_{0.05} / \Lambda=120,300,500,1000,3000$ corresponding to $0.05 \mathrm{Mpc}^{-1}$. The $z=3000$ curve almost overlaps with that of the $\Lambda \mathrm{CDM}$ model with a scale-invariant power spectrum. We normalize all the anisotropy spectra at the first Doppler peak. Also shown are the three-year WMAP data including error bars.

FIG. 3 (a) B0.1A7

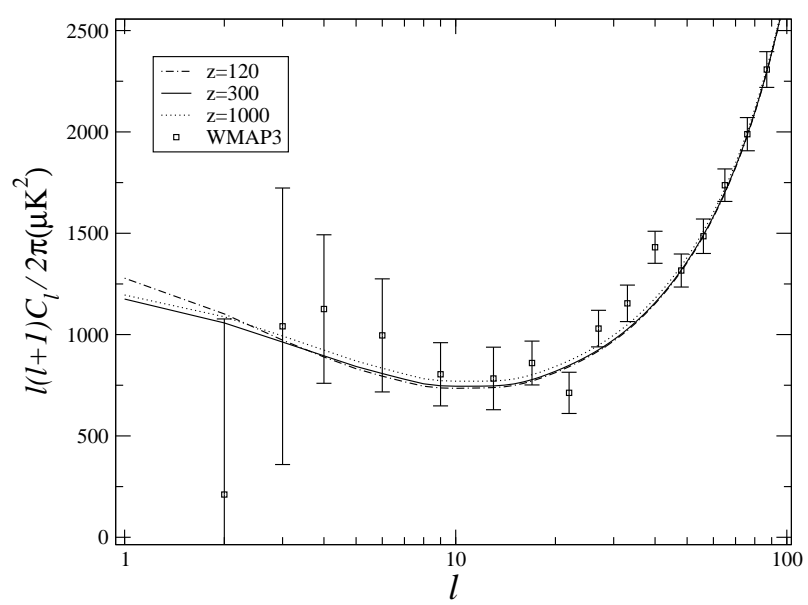

FIG. 3 (b) B0.1A5

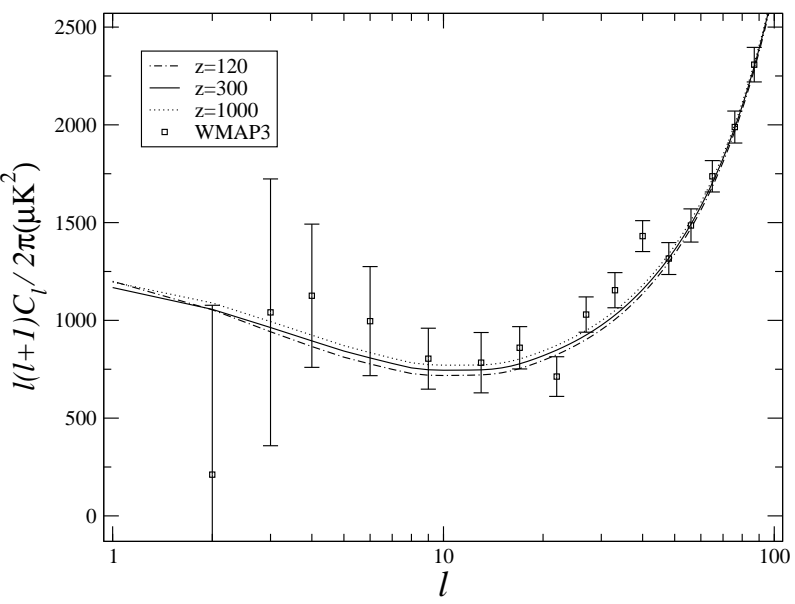


FIG. 3 (c) B0.1A1

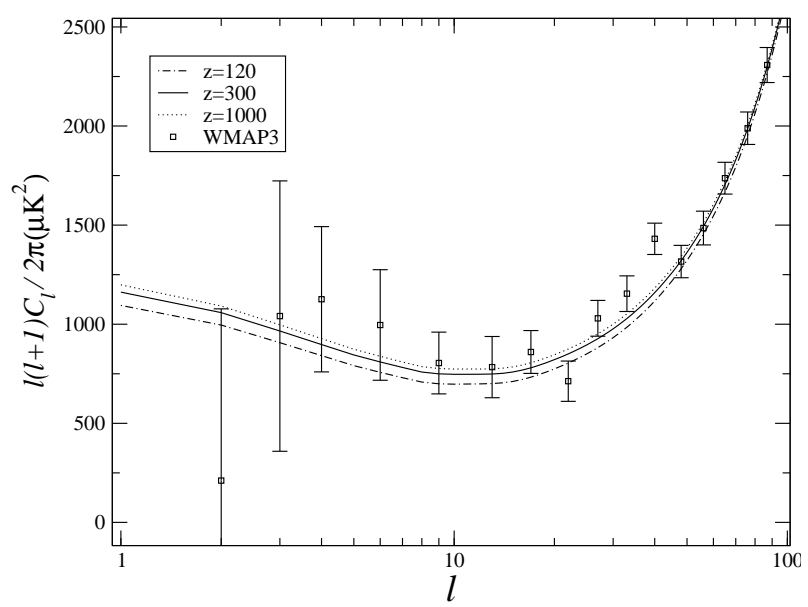

FIG. 3 (d) $\quad$ B0.1A0.1

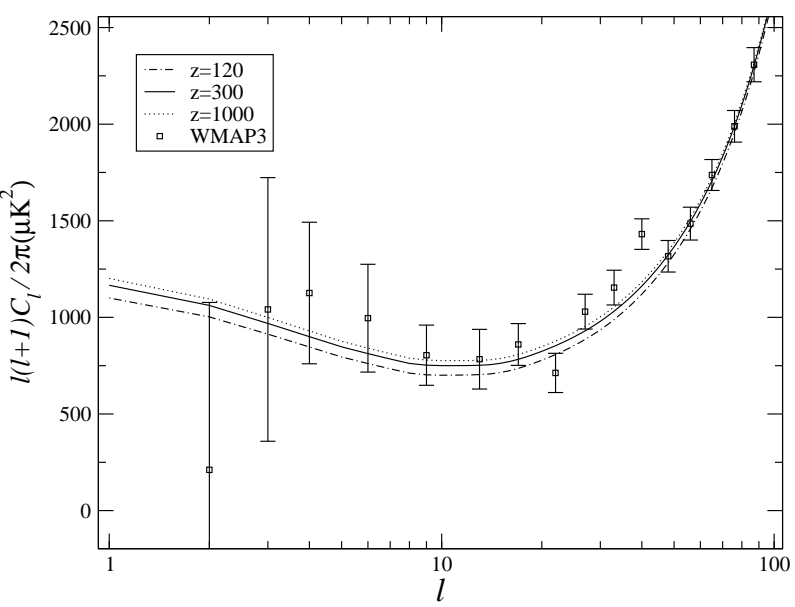

FIG. 3: The same as in Fig. 2 but for $B=0.1$ and $A=7,5,1,0.1$. 